\title{
Economic Load Dispatch Using a Chemotactic Differential Evolution Algorithm
}

\author{
Arijit Biswas ${ }^{1}$, Sambarta Dasgupta ${ }^{1}$, Bijaya K Panigrahi ${ }^{2}$, V. Ravikumar Pandi ${ }^{2}$, \\ Swagatam Das ${ }^{2}$, Ajith Abraham ${ }^{3}$ and Youakim Badr ${ }^{3}$ \\ ${ }^{1}$ Dept. of Electronics and Telecommunication Engg, \\ Jadavpur University, Kolkata, India \\ ${ }^{2}$ Department of Electrical Engineering, IIT, Delhi, India \\ ${ }^{3}$ National Institute of Applied Sciences of Lyon, INSA-Lyon, Villeurbanne, France \\ bkpanigrahi@ee.iitd.ac.in, swagatamdas19@yahoo.co.in, \\ ajith.abrahamaieee.org, youakim.badreinsa-lyon.fr
}

\begin{abstract}
This paper presents a novel stochastic optimization approach to solve constrained economic load dispatch (ELD) problem using Hybrid Bacterial Foraging-Differential Evolution optimization algorithm. In this hybrid approach computational chemotaxis of BFOA, which may also be viewed as a stochastic gradient search, has been coupled with DE type mutation and crossover of the optimization agents. The proposed methodology easily takes care of solving non-convex economic load dispatch problems along with different constraints like transmission losses, dynamic operation constraints (ramp rate limits) and prohibited operating zones. Simulations were performed over various standard test systems with different number of generating units and comparisons are performed with other existing relevant approaches. The findings affirmed the robustness and proficiency of the proposed methodology over other existing techniques.
\end{abstract}

\section{Introduction}

Economic load dispatch (ELD) problem [1,2] is a constrained optimization problem in power systems that have the objective of dividing the total power demand among the online participating generators economically while satisfying the various constraints. Over the years, many efforts have been made to solve the problem, incorporating different kinds of constraints or multiple objectives, through various mathematical programming and optimization techniques. The conventional methods include Lambda iteration method [3, 4], base point and participation factors method $[3,4]$, gradient method [3,5], etc. Among these methods, lambda iteration is most common one and, owing to its ease of implementation, has been applied through various software packages to solve ELD problems. But for effective implementation of this method, the formulation needs to be continuous. The basic ELD considers the power balance constraint apart from the generating capacity limits. However, a practical ELD must take ramp rate limits, prohibited operating zones, valve point loading effects, and multi fuel options [6] into consideration to provide the completeness for the ELD problem formulation. The resulting ELD is a non-convex optimization problem, which is a challenging one and cannot be solved by the traditional methods. An ELD problem with valve point loading has also been solved by dynamic programming (DP) [7,8]. Though promising results are obtained in small sized power systems while solving it with DP, it unnecessarily raises the length of 
solution procedure resulting in its vulnerability to solve large size ELD problems in stipulated time frames.

Moreover, evolutionary and behavioral random search algorithms such as Genetic Algorithm (GA) [9 - 11], Particle Swarm Optimization (PSO) [12, 13] etc. have previously been implemented on the ELD problem at hand. In addition, an integrated parallel GA incorporating ideas form simulated annealing (SA) and Tabu search (TS) techniques was also proposed in [14] utilizing generator's output power as the encoded parameter. Yalcinoz has used a real-coded representation technique along with arithmetic genetic operators and elitistic selection to yield a quality solution [15]. GA has been deployed to solve ELD with various modifications over the years. In a similar attempt, a unit independent encoding scheme has also been proposed based on equal incremental cost criterion [16]. In spite of its successful implementation, GA does posses some weaknesses leading to longer computation time and less guaranteed convergence, particularly in case of epistatic objective function containing highly correlated parameters $[17,18]$.

This paper proposes a new optimization approach, to solve the ELD using a hybrid Bacterial Foraging (BF) [19] -Differential Evolution (DE) [20, 21] algorithm, which is a recently emerged stochastic optimization technique. Passino proposed the Bacterial Foraging optimization technique, where the social foraging behavior of Escherichia coli (those living in our intestines) has been studied thoroughly. On the other hand DE is a simple Genetic Algorithm (GA) [22], which implements a differential mutation operator that distinguishes it from traditional GA. In this work the chemotaxis step of bacterial foraging is made adaptive and merged with the DE in order to tackle real world problems in a more elegant way.

\section{Problem description}

In a power system, the unit commitment problem has various sub-problems varying from linear programming problems to complex non-linear problems. The concerned problem, i.e., Economic Load Dispatch (ELD) problem is one of the different nonlinear programming sub-problems of unit commitment.

\section{Problem description}

In a power system, the unit commitment problem has various sub-problems varying from linear programming problems to complex non-linear problems. The concerned problem, i.e., Economic Load Dispatch (ELD) problem is one of the different non-linear programming sub-problems of unit commitment. The ELD problem is about minimizing the fuel cost of generating units for a specific period of operation so as to accomplish optimal generation dispatch among operating units and in return satisfying the system load demand, generator operation constraints with ramp rate limits and prohibited operating zones. The ELD problem with smooth and nonsmooth cost functions is considered in this paper.

\subsection{ELD problem formulation}

The objective function corresponding to the production cost can be approximated to be a quadratic function of the active power outputs from the generating units. Symbolically, it is represented as 
Minimize $\mathrm{F}_{\mathrm{t}}^{\cos \mathrm{t}}=\sum_{\mathrm{i}=1}^{\mathrm{N}_{\mathrm{G}}} f_{\mathrm{i}}\left(\mathrm{P}_{\mathrm{i}}\right)$

where $f_{\mathrm{i}}\left(\mathrm{P}_{\mathrm{i}}\right)=\mathrm{a}_{\mathrm{i}} \mathrm{P}_{\mathrm{i}}^{2}+\mathrm{b}_{\mathrm{i}} \mathrm{P}_{\mathrm{i}}+\mathrm{c}_{\mathrm{i}}, \quad \mathrm{i}=1,2,3, \ldots, \mathrm{N}_{\mathrm{G}}$

is the expression for cost function corresponding to $i^{\text {th }}$ generating unit and $a_{i}, b_{i}$ and $c_{i}$ are its cost coefficients. $P_{i}$ is the real power output (MW) of $i^{\text {th }}$ generator corresponding to time period t. $\mathrm{N}_{\mathrm{G}}$ is the number of online generating units to be dispatched. This constrained ELD problem is subjected to a variety of constraints depending upon assumptions and practical implications. These include power balance constraints to take into account the energy balance; ramp rate limits to incorporate dynamic nature of ELD problem and prohibited operating zones. These constraints are discussed as under.

1) Power Balance Constraints or Demand Constraints:

This constraint is based on the principle of equilibrium between total system

generation $\left(\sum_{i=1}^{N_{G}} P_{i}\right)$ and total system loads $\left(P_{D}\right)$ and losses $\left(P_{L}\right)$. That is,

$$
\sum_{i=1}^{N_{G}} P_{i}=P_{D}+P_{L}
$$

where $\mathrm{P}_{\mathrm{L}}$ is obtained using $\mathrm{B}$ - coefficients, given by

$$
\mathrm{P}_{\mathrm{L}}=\sum_{\mathrm{i}=1}^{\mathrm{N}_{\mathrm{G}}} \sum_{\mathrm{j}=1}^{\mathrm{N}_{\mathrm{G}}} \mathrm{P}_{\mathrm{i}} \mathrm{B}_{\mathrm{ij}} \mathrm{P}_{\mathrm{j}}
$$

2) The Generator Constraints: The output power of each generating unit has a lower and upper bound so that it lies in between these bounds. This constraint is represented by a pair of inequality constraints as follows.

$$
\mathrm{P}_{\mathrm{i}}^{\min } \leq \mathrm{P}_{\mathrm{i}} \leq \mathrm{P}_{\mathrm{i}}^{\max }
$$

where, $\mathrm{P}_{\mathrm{i}}^{\mathrm{min}}$ and $\mathrm{P}_{\mathrm{i}}^{\mathrm{max}}$ are lower and upper bounds for power outputs of the $\mathrm{i}^{\text {th }}$ generating unit.

3) The Ramp Rate Limits: One of unpractical assumption that prevailed for simplifying the problem in many of the earlier research is that the adjustments of the power output are instantaneous. However, under practical circumstances ramp rate limit restricts the operating range of all the online units for adjusting the generator operation between two operating periods. The generation may increase or decrease with corresponding upper and downward ramp rate limits. So, units are constrained due to these ramp rate limits as mentioned below.

If power generation increases, $\mathrm{P}_{\mathrm{i}}-\mathrm{P}_{\mathrm{i}}^{\mathrm{t}-1} \leq \mathrm{UR}_{\mathrm{i}}$

If power generation decreases, $\mathrm{P}_{\mathrm{i}}^{\mathrm{t}-1}-\mathrm{P}_{\mathrm{i}} \leq \mathrm{DR}_{\mathrm{i}}$

where $P_{i}^{t-1}$ is the power generation of unit $i$ at previous hour and $U_{i}$ and $D_{i}$ are the upper and lower ramp rate limits respectively. The inclusion of ramp rate limits modifies the generator operation constraints (5) as follows.

$$
\max \left(\mathrm{P}_{\mathrm{i}}^{\min }, \mathrm{UR}_{\mathrm{i}}-\mathrm{P}_{\mathrm{i}}\right) \leq \mathrm{P}_{\mathrm{i}} \leq \min \left(\mathrm{P}_{\mathrm{i}}^{\max }, \mathrm{P}_{\mathrm{i}}^{\mathrm{t}-1}-\mathrm{DR}_{\mathrm{i}}\right)
$$

4) Prohibited Operating Zone: The generating units may have certain ranges where operation is restricted on the grounds of physical limitations of machine components or instability e.g. due to steam valve or vibration in shaft bearings. Consequently, discontinuities are produced in cost curves corresponding to the prohibited operating 
zones. So, there is a quest to avoid operation in these zones in order to economize the production. Symbolically, for a generating unit $i$,

$$
\mathrm{P}_{\mathrm{i}} \leq \breve{\mathrm{P}}^{\mathrm{pz}} \text { and } \mathrm{P}_{\mathrm{i}} \geq \widehat{\mathrm{P}}^{\mathrm{pz}}
$$

where $\breve{\mathrm{P}}^{\mathrm{pz}}$ and $\widehat{\mathrm{P}}^{\mathrm{pz}}$ are the lower and upper are limits of a given prohibited zone for generating unit $i$.

\subsection{ELD constraints handling}

The equality and inequality constraints of the ELD problem are considered in the Fitness function (Jerror) itself by incorporating a penalty function

$$
\mathrm{PF}_{\mathrm{i}}=\left\{\begin{array}{c}
\mathrm{k}_{\mathrm{i}}\left(\mathrm{U}_{\mathrm{i}}-\mathrm{U}_{\mathrm{i}}^{\lim }\right)^{2} \text { if violated } \\
0 \quad \text { otherwise }
\end{array}\right.
$$

Where $\mathrm{k}_{\mathrm{i}}$ is the constant, called penalty factor for the $i^{\text {th }}$ constraint. Now the final solution should not contain any penalty for the constraint violation. Therefore the objective of the problem is the minimization of generation cost and penalty function due to any constraint violation as defined by the following equation

$$
\mathrm{J}_{\text {error }}=\mathrm{F}_{\mathrm{t}}^{\cos \mathrm{t}}+\sum_{\mathrm{i}=1}^{\mathrm{nc}} \mathrm{PF} \text {, where "nc" is the number of constraints. }
$$

\section{The Hybrid Algorithm}

DE has reportedly outperformed powerful meta-heuristics like genetic algorithm (GA) and particle swarm optimization (PSO) [23]. Practical experiences suggest that DE may occasionally stop proceeding towards the global optima, while the population has not converged to a local optima or any other point. Occasionally even new individuals may enter the population but the algorithm does not progress by finding any better solutions. This situation is usually referred to as stagnation [24]. In the present work, we have incorporated an adaptive chemotactic step borrowed from the realm of BFOA into DE. The computational chemotaxis in BFOA serves as a stochastic gradient descent based local search .It was seen to greatly improvise the convergence characteristics of the classical DE. The resulting hybrid algorithm is referred here as the CDE (Chemotactic Differential Evolution).

\section{The CDE (Chemotactic DE) Algorithm:}

Initialize parameters $S, N_{C}, N_{S}, C(i)(i=1,2 \ldots N), F, C R$.

where,

$S$ : The number of bacteria in the population,

D:Dimension,

$N_{C}$ : No. of chemotactic steps,

$C(i)$ : the size of the step taken in the random direction specified by the tumble.

$F$ : Scale factor for DE type mutation

$C R$ : Crossover Rate.

Set $j=0, t=0$;

Chemotaxis loop: $j=j+1$; 
Differential evolution mutation loop: $t=t+1$;

$\theta(i, j, t)$ denotes the position of the ith bacterium in the jth chemotactic and $\mathrm{t}$ th differential evolution loop.

for $i=1,2, \ldots \ldots, S$, a chemotactic step is taken for i-th bacterium.

(a)Chemotaxis loop:

(i) Value of the objective function $J(i, j, t)$ is computed, where $J(i, j, t)$ symbolizes value of objective function at $\mathrm{j}$ th chemotaxis cycle for $\mathrm{i}$ - th bacterium at $\mathrm{t}$-th $\mathrm{DE}$ mutation step;

(ii) $J_{\text {last }}=J(i, j, t)$ we store this value of objective function for comparison with values of objective function yet to be obtained in future.

(iii) Tumble: generate a random vector $\Delta(i) \in \mathfrak{R}^{D}$ with each element $\Delta_{m}(i), m=1,2, \ldots \ldots, D$ is a random number on $[-1,1]$.

(iv) Move: $\theta(i, j+1, t)=\omega \cdot \theta(i, j, t)+C(i) \cdot\left(\Delta(i) / \sqrt{\Delta(i) \cdot \Delta^{T}(i)}\right)$;

Where, $\omega=$ inertia factor which is generally equals to 1 but becomes 0.8 if the function has an optimal value close to 0 .

$$
C(i)=\text { step size for } \mathrm{k} \text { th bacterium }=0.1 \cdot \frac{J(i, j, t))}{(J(i, j, t)+1000)}
$$

Step size is made an increasing function of objective function value to have a feedback arrangement.

(v) $J(i, j, t)$ is computed.

(vi) Swim: We consider here only $i$-th bacterium is moving and others are not moving. Now Let $m=0$;

while $m<N_{s}$ (no of steps less than max limit).

Let $m=m+1$;

If $\quad J(i, j, t)<J_{\text {last }}$ (if going better)

$$
J_{\text {last }}=J(i, j, t) \text {; }
$$

And let, $\theta(i, j+1, t)=\omega \cdot \theta(i, j, t)+C(i) \cdot\left(\Delta(i) / \sqrt{\Delta(i) \cdot \Delta^{T}(i)}\right)$

Else, $m=N_{s}$ (end of while loop);

for $i=1,2, \ldots \ldots, S$, a differential evolution mutation step is taken for $\mathrm{i}$-th bacterium.

\section{(b) Differential Evolution Mutation Loop:}

(i) For each $\theta(i, j+1, t)$ trial solution vector we choose randomly three other distinct vectors from the current population namely $\theta(l), \theta(m), \theta(n)$ such that

$$
i \neq l \neq m \neq n
$$

(ii) $V(i, j+1, t)=\theta(l)+F .(\theta(m)-\theta(n))$;

Where, $V(i, j+1, t)$ is the donor vector corresponding to $\theta(i, j+1, t)$.

(iii) Then the donor and the target vector interchange components probabilistically to yield a trial vector $U(i, j+1, t)$ following:

$$
\begin{aligned}
U_{p}(i, j+1, t)= & V_{p}(i, j+1, t) \text { If }\left(\operatorname{rand}_{p}(0,1) \leq C R\right) \text { or }(p=r n(i)) \\
& \theta_{p}(i, j+1, t) \text { If }\left(\operatorname{rand}_{p}(0,1)>C R\right) \text { or }(p \neq r n(i)) \text { for } \mathrm{p} \text {-th }
\end{aligned}
$$

dimension 
where $\operatorname{rand}_{\mathrm{p}}(0,1) \in[0,1]$ is the $\mathrm{p}$-th evaluation of a uniform random number generator. $r n(i) \in\{1,2, \ldots, D\}$ is a randomly chosen index which ensures that $U(i, j+1, t)$ gets at least one component from $V(i, j+1, t)$.

(iv) $J(i, j+1, t)$ is computed for trial vector;

(v) If $J(U(i, j+1, t))<J(\theta(i, j+1, t)), \theta(i, j+1, t+1)=U(i, j+1, t)$;

Original vector is replaced by offspring if value of objective function for it is smaller.

If $j<N_{c}$, start another chemotaxis loop.

\section{Experiment Results and Discussions}

\subsection{ELD with Smooth and Non Smooth Cost Function considering Ramp Rate Limits and Prohibited Operating Zones}

The applicability and viability of the aforementioned technique for practical applications has been tested on four different power system cases. The obtained results are compared with the reported results of GA, PSO [12], CPSO [25], PSOLRS, NPSO and NPSO-LRS [26] and Chaotic Differential Evolution [27, 28] methods. The cases taken for our study comprises of $6,13,15$ and 40 generator systems. Following subsections deal with the detailed discussion of the obtained results.

\subsection{Six-Unit System}

The system contains six thermal generating units. The total load demand on the system is $1263 \mathrm{MW}$. The results are compared with the elitist GA [12], PSO [12], NPSO-LRS [26] and CPSO [25] methods for this test system. Parameters of all the thermal units are reported in [12]. Results obtained using the proposed hybridized Bacterial Foraging algorithm is listed in table 1 . It can be evidently seen from table 1 that the technique provided better results compared to other reported evolutionary algorithm techniques. It is also observed that the minimum cost using the proposed approach is less than the reported minimum cost using some of other methods. The standard deviation of the cost is 0.0147 \$.

\subsection{Thirteen-Unit System}

This test system consists of 13 generating units with valve point loading as mentioned in [29]. The expected load demand for this system is $1800 \mathrm{MW}$. Since this is larger system with more nonlinearities, it has more local minima and difficult to obtain the global solution. The best result obtained is reported in Table 2 and compared with other recently reported results. Another reported result for minimum cost in [29] is \$17994.07. 
Table I: Result for a six-unit system for demand of $1263 \mathrm{MW}$

\begin{tabular}{|c|c|c|c|c|c|}
\hline Generator Power Output (MW) & BF_DE Hybrid & PSO[12] & GA[12] & $\begin{array}{c}\text { NPSO-LRS } \\
{[34]}\end{array}$ & CPSO1 [33] \\
\hline $\mathrm{P}_{\mathrm{G} 1}$ & 446.7146 & 447.4970 & 474.8066 & 446.96 & 434.4236 \\
\hline $\mathrm{P}_{\mathrm{G} 2}$ & 173.1485 & 173.3221 & 178.6363 & 173.3944 & 173.4385 \\
\hline $\mathrm{P}_{\mathrm{G} 3}$ & 262.7945 & 263.4745 & 262.2089 & 262.3436 & 274.2247 \\
\hline $\mathrm{P}_{\mathrm{G} 4}$ & 143.4884 & 139.0594 & 134.2826 & 139.5120 & 128.0183 \\
\hline $\mathrm{P}_{\mathrm{G} 5}$ & 163.9163 & 165.4761 & 151.9039 & 164.7089 & 179.7042 \\
\hline $\mathrm{P}_{\mathrm{G} 6}$ & 85.3553 & 87.1280 & 74.1812 & 89.0162 & 85.9082 \\
\hline Total Power Generation $(\mathrm{MW})$ & 1275.4 & 1276.01 & 1276.03 & 1275.94 & 1276.0 \\
\hline Minimum Cost $(\$ / \mathrm{hr})$ & 15444.1564 & 15450 & 15459 & 15450 & 15447 \\
\hline Ploss $(\mathrm{MW})$ & 12.4220 & 12.9584 & 13.0217 & 12.9361 & 12.9583 \\
\hline Mean Cost $(\$ / \mathrm{hr})$ & 15444.7815 & 15454 & 15469 & 15450.5 & 15449 \\
\hline Standard Deviation of Cost $(\$ / \mathrm{hr})$ & 0.0147 & - & - & - & - \\
\hline
\end{tabular}

-: Not reported in the referred literature.

Table II: Result for a 13-unit system for a demand of $1800 \mathrm{MW}$.

\begin{tabular}{|c|c|c|}
\hline Generator Power output (MW) & $\begin{array}{c}\text { Bacterial Foraging Differential } \\
\text { Evolution Hybrid }\end{array}$ & $\begin{array}{c}\text { Chaotic Differential } \\
\text { Evolution [36] }\end{array}$ \\
\hline $\mathrm{P}_{\mathrm{G} 1}$ & 628.3185306858442 & 628.3173 \\
\hline $\mathrm{P}_{\mathrm{G} 2}$ & 149.59965011285834 & 149.2407 \\
\hline $\mathrm{P}_{\mathrm{G} 3}$ & 222.753309362145 & 109.8540 \\
\hline $\mathrm{P}_{\mathrm{G} 4}$ & 109.86655008717487 & 109.8657 \\
\hline $\mathrm{P}_{\mathrm{G} 5}$ & 109.86327261039418 & 109.8666 \\
\hline $\mathrm{P}_{\mathrm{G} 6}$ & 109.86654988406237 & 109.8211 \\
\hline $\mathrm{P}_{\mathrm{G} 7}$ & 109.86337243612016 & 109.8664 \\
\hline $\mathrm{P}_{\mathrm{G} 8}$ & 109.86654836418003 & 60.000 \\
\hline $\mathrm{P}_{\mathrm{G} 9}$ & 59.99957824230915 & 40.000 \\
\hline $\mathrm{P}_{\mathrm{G} 10}$ & 39.999657552894476 & 40.000 \\
\hline $\mathrm{P}_{\mathrm{G} 11}$ & 39.997977001623795 & 55.000 \\
\hline $\mathrm{P}_{\mathrm{G} 12}$ & 54.99916355936233 & 55.000 \\
\hline $\mathrm{P}_{\mathrm{G} 13}$ & 54.999507665171905 & 1800.00 \\
\hline Total Power Generation $(\mathrm{MW})$ & 1799.99 & 17963.9401 \\
\hline Minimum Cost $(\$ / \mathrm{hr})$ & 17960.3966 & 17973.1339 \\
\hline Mean Cost $(\$ / \mathrm{hr})$ & 17960.6258 & 1.9735 \\
\hline Standard Deviation of Cost $(\$ / \mathrm{hr})$ & 0.1371 & \\
\hline & & \\
\hline
\end{tabular}

\section{Conclusions}

The paper has employed the hybridized bacterial foraging-differential evolution algorithm on the constrained economic load dispatch problem. Practical generator operation is modeled using several non linear characteristics like ramp rate limits, prohibited operating zones. The proposed approach has produced results comparable or better than those generated by other evolutionary algorithms and the solutions obtained have superior solution quality and good convergence characteristics. From this limited comparative study, it can be concluded that the applied algorithm can be effectively used to solve smooth as well as non-smooth constrained ELD problems. In future, efforts will be made to incorporate more realistic constraints to the problem structure and the practical large sized problems would be attempted by the proposed methodology.

\section{References}

[1]. B.H Choudhary, and S. Rahman, "A Review of recent advances in economic dispatch," IEEE Trans. on Power System, Vol. 5, No. 4, pp. 1248-1259, Nov. 1990. 
[2]. H.H. Happ, "Optimal power dispatch-a comprehensive survey," IEEE Trans on Power Apparatus and Systems, Vol. PAS-96, pp. 841-854, May/June, 1971.

[3]. A.J. Wood and B.F. Wollenberg, Power Generation, Operation and Control, John Wiley \& Sons, New York, 1984.

[4]. C.L. Chen, and S.C. Wang, "Branch and bound scheduling for thermal generating units," IEEE Trans. on Energy Conversion, Vol. 8, No. 2, pp. 184-189, June 1993.

[5]. K.Y. Lee, et al., "Fuel cost minimization for both real and reactive power dispatches," IEE Proc. C, Gener. Trsns. \& distr., 131, (3), pp. 85-93, 1984.

[6]. C.E. Lin, and G.L. Viviani, "Hierarchical economic dispatch for piecewise quadratic cost functions," IEEE Trans. on Power Apparatus Systems, Vol. PAS103, pp.1170-1175, June1984.

[7]. A. Bakirtzis, V. Petridis, and S. Kazarlis, "Genetic algorithm solution to the economic dispatch problem,” Proc. Inst. Elect. Eng. Gen., Transm. Distrib., vol. 141, no. 4, July, 1994, pp. 377-382.

[8]. F.N. Lee, and A.M. Breipohl, "Reserve constrained economic dispatch with prohibited operating zones," IEEE Trans. Power Syst. 8 (February) (1993), 246-254.

[9]. G.B. Sheble and K. Brittig, "Refined genetic algorithm-economic dispatch example," IEEE Paper 94 WM 199-0 PWRS, presented at the IEEE/PES 1994 Winter Meeting.

[10]. D.C. Walters and G.B. Sheble, "Genetic algorithm solution of economic dispatch with valve point loading," IEEE Trans. on Power Systems, Vol. 8, NO. 3, pp. 1325-1332, August 1993.

[11]. A.A, Ma, El-Keib and R.E. Smith, "A genetic algorithm-based approach to economic dispatch of power systems," IEEE conference, 1994.

[12]. Zwe-Lee Gaing, "Particle swarm optimization to solving the economic dispatch considering the generator constraints," IEEE Trans. on Power Systems, Vol. 18, No. 3, pp., 1187-1195, August 2003.

[13]. J. B. Park, K. S. Lee, J. R. Shin, and K. Y. Lee. "A Particle Swarm optimization for economic dispatch with non-smooth cost functions," IEEE Transactions on Power Systems, Vol. 20, No. 1, Feb. 2005, pp. 34-42.

[14]. P.H. Chen, and H.C. Chang, "Large scale economic dispatch by genetic algorithm," IEEE Trans. Power Syst. 10 (4), 1995, pp.1919-1926.

[15]. T. Yalcionoz, H. Altun, and M. Uzam, "Economic dispatch solution using a genetic algorithm based on arithmetic crossover," in Proc. IEEE Proto Power Tech. Conf., Proto, Portugal, Sept. 2001.

[16]. C. C. Fung, S. Y. Chow, and K. P. Wong, "Solving the economic dispatch problem with an integrated parallel genetic algorithm," in Proc. PowerCon Int. Conf., Vol. 3, 2000, pp. 1257-1262.

[17]. D.B. Fogel, Evolutionary Computation: Toward a New Philosophy of Machine Intelligence, second ed., IEEE Press, Piscataway, NJ, 2000.

[18]. R.C. Eberhart, and Y. Shi, "Comparison between genetic algorithms and particle swarm optimization,” Proc. IEEE Int. Conf. Evol. Comput., May, 1998, pp. 611-616.

[19]. K. M. Passino, "Biomimicry of bacterial foraging for distributed optimization and control," IEEE. Control Syst. Mag., pp. 52-67, June. 2002.

[20]. K. Price, R. Storn, and J. Lampinen, Differential Evolution - A Practical Approach to Global Optimization, Springer, ISBN: 3-540-20950-6, 2005.

[21]. A. Biswas, S. Dasgupta, S. Das, and A. Abraham, A Synergy of Differential Evolution and Bacterial Foraging Algorithm for Global Optimization, International Journal on Neural and Mass-Parallel Computing and Information Systems, Neural Network World, Volume 17, No. 6, pp. 607-626, 2007.

[22]. D. E. Goldberg, Genetic Algorithms in Search, Optimization and Machine Learning, Addison-Wesley, Reading, MA, 1989.

[23]. J. Vesterstrøm and R. Thomson: A Comparative Study of Differential Evolution, Particle Swarm Optimization, and Evolutionary Algorithms on Numerical Benchmark Problems, Proc. Sixth Congress on Evolutionary Computation (CEC-2004). IEEE Press.

[24]. Lampinen, J., Zelinka, I.: On Stagnation of the Differential Evolution Algorithm, In: Ošmera, Pavel (ed.) (2000). Proceedings of MENDEL 2000, 6th International Mendel Conference on Soft Computing,June 7.-9. 2000, Brno, Czech Republic.

[25]. C. Jiejin, M. Xiaoqian, L. Lixiang and P. Haipeng, "Chaotic particle swarm optimization for economic dispatch considering the generator constraints," Energy conver. and Management, vol. 48, 2007, pp. 645-653.

[26]. A. Immanuel Selvakumar and K. Thanushkodi , "A new particle swarm optimization Solution to nonconvex economic dispatch problems," IEEE Trans. on power systems, Vol. 22, No. 1, Feb 2007, pp. $42-51$.

[27]. L. D. S. Coelho and V. C. Mariani, "Combining of chaotic differential evolution and quadratic programming for economic dispatch optimization with valve point effect," IEEE Trans. on Power systems, Vol. 21, no.2, pp. 989-996, May 2006.

[28]. L. D. S. Coelho and V. C. Mariani, "Erratum Correlation to - Combining of chaotic differential evolution and quadratic programming for economic dispatch optimization with valve point effect," IEEE Trans. on Power systems, Vol. 21, no.3, pp. 1465, August 2006.

[29]. N. Sinha, R. Chakrabarti, and P. K. Chattopadhyay, "Evolutionary programming techniques for economic load dispatch," IEEE Trans. Evol. Comput., vol. 7, no. 1, pp. 83-94, Feb. 2003. 